\title{
SOME RESULTS ON BOUNDARY VALUE PROBLEMS FOR FUNCTIONAL DIFFERENTIAL EQUATIONS
}

\section{P. CH. TSAMATOS and S. K. NTOUYAS}

University of Ioannina

Department of Mathematics

45110 Ioannina, Gineres

(Received July 15, 1993 and in revised form December 29, 1993)

\begin{abstract}
ABSTRAC'T. Existence results for a second order boundary value problem for functional differential equations, are given. The results are based on the nonlinear Alternative of Leray-Schauder and rely .on a priori bounds on solutions. These results are generalizations of recent results from ordinary differential equations and complete our earlier results on the same problem.
\end{abstract}

KEY WORDS AND PHRASES. Boundary value problems, functional differential equations,a priori bounds, Leray-Schander alternative. 1992 AMS SUBJECT CLASSIFICATION CODES. $34 \mathrm{~K} 10$

\section{INTRODUC'TION.}

The purpose of this paper is to provide existence results for second order boundary value problems (BVP for short) for functional differential equations. Before we refer our BVP, for the convenience of the reader, we employ the standar setting for functional differential equations, [3].

Let $r \geq 0$ be given and let $C=C\left([-r, 0], R^{n}\right)$ denote the space of continuous functions that map the interval $[-r, 0]$ into $R^{n}$. For $\phi \in C$, the norm of $\phi$ is defined by

$$
|\phi|=\sup \{|\phi(\theta)|: \quad-r \leq \theta \leq 0\}
$$

where $|$.$| denotes any convenient norm in R^{n}$. If $x:[-r, T] \rightarrow R^{n}, T>0$ is continuous, then for each $t \in[0, T], x_{t} \in C$ is defined by $x_{t}(\theta)=x(t+\theta),-r \leq \theta \leq 0$.

We consider the following BVP

$$
\begin{gathered}
x^{\prime \prime}(t)+f\left(t, x_{t}, x^{\prime}(t)\right)=0, \quad t \in[0, T] \\
\alpha_{0} x_{0}-\alpha_{1} x^{\prime}(0)=\emptyset \\
\beta_{0} x(T)+\beta_{1} x^{\prime}(T)=\eta
\end{gathered}
$$

where $f:[0, T] \times C \times R^{n} \rightarrow R^{n}$ is a continuous function, $\phi \in C, \eta \in R^{n}$ and $a_{0}, a_{1}, \beta_{0}, \beta_{1}$ are positive real constants. 
At first glanes it might appear to the reader that the tirst boundary condition in (BC) is an

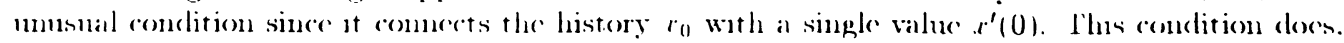
howeren, arese in a natural way and is suggested hy the well posedness of the BDP (E)-(BC'), since the function $f$ depends on the term $r$ and simultaneonsly. on the values of derivatives at the porsent time.

It should be noted that the BTP (E)-(BC') has been studied carlice in $H$ H under narions condition on $f$.

The basic existene thenem for the BIP (E)-(BC'), which relies on the a prion bounds, has been proved recently in $[4]$ and it is not given here. He cmphasize here that in the proof of the existenere theorem in [4] we have not used the standand ansmuption that f maps bounded sets of $\left[0 . I^{\prime}\right] \times C^{\prime} \times R^{n}$ into bounded sets in $R^{n}$.

Our main purpose in this paper is to give conditions on $f$ wich imply the neded a prion bounds. These conditions ane different from those given in [H]. More precisely, the a promi bomels for the solutions $x$ and its derivatives $r^{\prime}$ are obtained via $L^{2}$-estimates( Sere $[i]$ ) and a Nagumo type condition analogous to that used in [2] for ordinary differential cequations.

The results of this paper are not comparable with those of $[4]$ and seems to be new even when

$(\mathrm{E})$ is an ordinary differential equation, i.e. $r=0$.

In what follows $\|$.$\| stands for the L^{2}$-norm defined by

$$
\|\cdot r\|=\left(\int_{0}^{T}|\cdot r(t)|^{2} d t\right)^{1 / 2}
$$

and $<\ldots>$ stads for the Eucliclean inner product in $R^{n}$.

For subsequent use we shall state here the following inequalitics.

LEMIMA 1.1. (a) For any function $r \in C^{\prime}[0, T]$

$$
\|\cdot r\| \leq \frac{2 T}{\pi}\left\|\cdot r^{\prime}\right\|+\frac{1}{2} \sqrt{T}|\cdot r(0)|+\frac{1}{2} \sqrt{T}|\cdot r(T)| .
$$

(b) For any function $x \in C^{\prime}[0 . T]$

$$
\|r\|^{2} \leq \frac{T^{2}}{\pi^{2}} \frac{2+\sqrt{2 T}}{2-T}\left\|x^{\prime}\right\|^{2}+\frac{\sqrt{T}}{\sqrt{2}}\left[|x(0)|^{2}+|x(T)|^{2}\right], \quad T=2 .
$$

The above inequalities follows by essentially the same reasoning as in lemmas 2.3 and 2.4 of [5]. Obviously in the case $T=2$ we can use the inequality (a) instead of (b).

\section{MAIN RESULTS}

Now we present our main result on the existence of solutions of the BVP (E)-(BC).

THEOREM 2.1. Let $f:[0, T] \times C \times R^{n} \rightarrow R^{n}$ be a continuous function. Assume that:

$$
\begin{aligned}
& \left(H_{1}\right) \text { There exist nonnegatıve constants } A \text { and } B \text { w'th } \\
& B<\min \left\{\left(1-\frac{A T^{2}}{\pi^{2}} \frac{2+\sqrt{2 T}}{2-T}\right) \frac{2 \pi}{4 T+\pi \sqrt{T}}, \frac{4}{\sqrt{T}}\left(\frac{\alpha_{0}}{a_{1}}-\frac{A \sqrt{T}}{\sqrt{2}}\right)\right. \\
& \left.\frac{4}{\sqrt{T}}\left(\frac{\beta_{0}}{3_{1}}-\frac{A \sqrt{T}}{\sqrt{2}}\right)\right\}
\end{aligned}
$$

such that

$$
<u(0), f(t, u, v)>\leq A|u(0)|^{2}+B|u(0) \| v|
$$

for all $t \in[0, T], u \in C$ and $v \in R^{n}$.

$\left(H_{2}\right)$ There exists a contenuous function $h: R^{+} \rightarrow R^{+}$and a constant 
$x$ such that

$$
\left\langle r f(t . u \cdot r)>\leq h\left(|\cdot|^{2}\right)|\cdot|^{2}\right.
$$

for all $t \in[0 . T] ., \in R^{n}$ and $u \in C$ with $|u| \leq M$ and

$$
\int_{\frac{y^{2}}{T}}^{x} \frac{d v}{\ln (-)} \geq 2 \mathrm{M}^{2}
$$

where

$$
\begin{aligned}
& M=\max \left\{\sqrt{k \cdot c^{-1}} \cdot \sqrt{\left.k \cdot c_{0}\right)^{-1}}+\frac{|\phi(0)|}{2 \alpha_{1} c_{0}} \cdot \sqrt{k \cdot c_{1}^{-1}}+\frac{|n|}{2 \cdot 3_{1} c_{1}}\right\} \\
& c=1-\frac{A T^{2}}{\pi^{2}} \frac{2+\sqrt{2 T}}{2-T}-\frac{2 B T}{\pi}-\frac{1}{2} B \sqrt{T} \\
& c_{0}=\frac{a_{0}}{a_{1}}-\frac{A \sqrt{T}}{\sqrt{2}}-\frac{1}{4} B \sqrt{T} \\
& c_{1}=\frac{\phi_{0}}{\xi_{1}}-\frac{A \sqrt{T}}{\sqrt{2}}-\frac{1}{4} B \sqrt{T} \\
& k=\frac{|\phi(0)|^{2}}{4 a_{0}()_{1}}+\frac{|\eta|^{2}}{43_{0} \cdot 3_{1}}
\end{aligned}
$$

Then the BVP (E)-(BC) has at least one solution.

PROOF. To prove the existence of solutions of the BVP (E)-(BC) we apply the Nonlinear Alternative of Leray-Schauder in the manner applied in [4]. To do this we need to verify that the set of all posible solutions of the family of $\mathrm{B} V P\left(E_{\lambda}\right)-(B C)$, where

$$
x^{\prime \prime}(t)+\lambda f\left(t, x_{t}, x^{\prime}(t)\right)=0, \quad t \in[0, T]
$$

is a priori bounded by a constant independent of $\lambda$.

Let $x$ be a solution of $\left(E_{\lambda}\right)-(B C)$. By taking the inner product of $\left(E_{\lambda}\right)$ with $x(t)$, intcgrating by parts over $[0, T]$ and using the fact that.

$$
\begin{aligned}
\int_{0}^{T}<x(t), x^{\prime \prime}(t)>d t & =<x(T), x^{\prime}(T)>-<x(0), x^{\prime}(0)>-\left\|x^{\prime}\right\|^{2} \\
& =<r(T), \frac{\eta-\beta_{0} x(T)}{\beta_{1}}>-<x(0), \frac{\alpha_{0} \cdot r(0)-\phi(0)}{a_{1}}>-\left\|\cdot r^{\prime}\right\|^{2} \\
& =<r(T), \frac{\eta}{\beta_{1}}>-\frac{\beta_{0}}{\beta_{1}}|x(T)|^{2}-\frac{\alpha_{0}}{a_{1}}|x(0)|^{2}+<r(0), \frac{\phi(0)}{a_{1}}>-\left\|\cdot r^{\prime}\right\|^{2} .
\end{aligned}
$$

we obtain by $\left(H_{1}\right)$

$$
\begin{aligned}
\left\|x^{\prime}\right\|^{2}+\frac{\alpha_{0}}{\alpha_{1}}|x(0)|^{2}+\frac{\beta_{0}}{3_{1}}|x(T)|^{2} & \leq \frac{|\phi(0)|}{\alpha_{1}}|x(0)|+\frac{|\eta|}{3_{1}}|x(T)|+\int_{0}^{T}<x(t), f\left(t, x_{t}, x^{\prime}(t)>d t\right. \\
& \leq \frac{|\phi(0)|}{a_{1}}|r(0)|+\frac{|\eta|}{3_{1}}|r(T)|+\int_{0}^{T}<r_{1}(0), f\left(t, r_{1}, r^{\prime}(t)>d t\right. \\
& \leq \frac{|\phi(0)|}{a_{1}}|x(0)|+\frac{|\eta|}{3_{1}}|x(T)|+A\|x\|^{2}+B\|x\|\left\|\cdot x^{\prime}\right\| .
\end{aligned}
$$


Lemma 1.1 implies

$$
\begin{aligned}
& \left\|x^{\prime}\right\|^{2}+\frac{\alpha_{0}}{\alpha_{1}}|x(0)|^{2}+\frac{3_{0}}{3_{1}}|x(T)|^{2} \\
& \leq \frac{|\phi(0)|}{a_{1}}|x(0)|+\frac{|n|}{3_{1}}|r(T)|+\frac{. t T^{2}}{\pi^{2}} \frac{2+\sqrt{2 T}}{2-T}\left\|r^{\prime}\right\|^{2} \\
& +\frac{A \sqrt{T}}{\sqrt{2}} \| r\left(\left.0\right|^{2}+|r(T)|^{2}\right] \\
& +B\left\|x^{\prime}\right\|\left\{\frac{2 T}{\pi}\left\|x^{\prime}\right\|+\frac{1}{2} \sqrt{T}|x(0)|+\frac{1}{2} \sqrt{T} \mid \cdot x(T) \|\right\} \\
& \leq \frac{|\phi(0)|}{a_{1}}|x(0)|+\frac{|\eta|}{3_{1}}|\cdot r(T)|+\frac{A T^{2}}{\pi^{2}} \frac{2+\sqrt{2 T}}{2-T}\left\|\cdot r^{\prime}\right\|^{\prime} \\
& +\frac{A \sqrt{T}}{\sqrt{2}}\left[|r(0)|^{2}+|\cdot r(T)|^{2}\right] \\
& +\frac{2 B T}{\pi}\left\|x^{\prime}\right\|^{2}+\frac{1}{2} B \sqrt{T}\left\{\frac{1}{2}|x(0)|^{2}+\frac{1}{2}\left\|x^{\prime}\right\|^{2}\right\} \\
& +\frac{1}{2} B \sqrt{T}\left\{\frac{1}{2}|x(T)|^{2}+\frac{1}{2}\left\|x^{\prime}\right\|^{2}\right\}
\end{aligned}
$$

Conşequently

$$
\begin{aligned}
\left(1-\frac{A T^{2}}{\pi^{2}}\right. & \left.\frac{2+\sqrt{2 T}}{2-T}-\frac{2 B T}{\pi}-\frac{1}{2} B \sqrt{T}\right)\left\|x^{\prime}\right\|^{2} \\
& +\left(\frac{\alpha_{0}}{\alpha_{1}}-\frac{A \sqrt{T}}{\sqrt{2}}-\frac{1}{4} B \sqrt{T}\right)|x(0)|^{2} \\
& +\left(\frac{\beta_{0}}{\beta_{1}}-\frac{A \sqrt{T}}{\sqrt{2}}-\frac{1}{4} B \sqrt{T}\right)|x(T)|^{2} \\
\leq & \frac{|\phi(0)|}{\alpha_{1}}|x(0)|+\frac{|\eta|}{\beta_{1}}|x(T)|
\end{aligned}
$$

or

$$
\begin{aligned}
c\left\|x^{\prime}\right\|^{2} & +\left(\sqrt{c_{0}}|x(0)|-\frac{|\phi(0)|}{2 \alpha_{1} \sqrt{c_{0}}}\right)^{2}+\left(\sqrt{c_{1}}|x(T)|-\frac{|\eta|}{2 \beta_{1} \sqrt{c_{1}}}\right)^{2} \\
& \leq \frac{|\phi(0)|^{2}}{4 \alpha_{0} \alpha_{1}}+\frac{|\eta|^{2}}{4 \beta_{0} \beta_{1}}=k .
\end{aligned}
$$

The last inequality implies that

$$
\left\|x^{\prime}\right\| \leq M, \quad|x(0)| \leq M, \quad \text { and } \quad|x(T)| \leq M
$$

Therefore for every $t \in[0, T]$

$$
\begin{aligned}
|x(t)| & \leq|x(0)|+\left|\int_{0}^{t} x^{\prime}(s) d s\right| \\
& \leq|x(0)|+\sqrt{T}\left\|x^{\prime}\right\| \leq M+\sqrt{T} M=(1+\sqrt{T}) M,
\end{aligned}
$$

which is the required a priori bound on $x$ on the interval $[0, T]$.

Next we shall prove that $x$ is bounded on $[-r, 0]$. From the first boundary condition we have:

$$
\alpha_{1}\left|x^{\prime}(0)\right| \leq|\phi(0)|+\alpha_{0} M \leq \mathbf{|} \phi \mid+\alpha_{0} M
$$

and consequently

$$
\boldsymbol{m} \leq \frac{1}{\alpha_{0}}\left\|\boldsymbol{\phi}+\alpha_{1}\left|\cdot x^{\prime}(0) \| \leq \frac{2}{\alpha_{0}}\right| \boldsymbol{\phi}+M .\right.
$$


Therefore

$$
\max r\{|r(t)|:-r \leq t \leq T\} \leq . M_{0}=\operatorname{mar}\left\{\frac{2}{a_{0}}|\boldsymbol{|}| . \mid M .(1+\sqrt{T} \mid . M\} .\right.
$$

Also, $(\star)$ implies, by the mean value theorem, that there cxists $t_{0} \in[0 . T]$ such that

$$
T\left|r^{\prime}\left(t_{0}\right)\right|^{2} \leq M^{2}
$$

or

$$
\left|r^{\prime}\left(t_{0}\right)\right|^{2} \leq \frac{M^{2}}{T}
$$

Now, taking the inner product of $\left(E_{\lambda}\right)$ with $x^{\prime}(t)$ we have by $\left(H_{2}\right)$

$$
\left.\left.\left|\frac{d}{d t}\right| r^{\prime}(t)\right|^{2}\left|\leq 2 h\left(\left|r^{\prime}(t)\right|^{2}\right)\right| r^{\prime}(t)\right|^{2}
$$

or

$$
\left|\frac{d}{d t} \int_{0}^{\left|r^{\prime}(t)\right|^{2}} \frac{d s}{h(s)}\right| \leq 2\left|x^{\prime}(t)\right|^{2}
$$

Integrating the above inequality we get.

$$
\begin{aligned}
& \int_{0}^{\left|r^{\prime}(t)\right|^{2}} \frac{d s}{h(s)} \leq \int_{0}^{\left|x^{\prime}\left(t_{0}\right)\right|^{2}} \frac{d s}{h(s)}+2 \int_{0}^{T}\left|r^{\prime}(t)\right|^{2} d t \\
& \leq \int_{0}^{\left|2^{\prime}\left(t_{0}\right)\right|^{2}} \frac{d s}{h(s)}+2 I^{2} \\
& \leq \int_{0}^{\frac{\omega^{2}}{T}} \frac{d s}{h(s)}+\int_{\frac{N^{2}}{T}}^{N} \frac{d s}{h(s)}=\int_{0}^{N} \frac{d s}{h(s)} \text {. }
\end{aligned}
$$

Hence

$$
\left|x^{\prime}(t)\right| \leq \sqrt{N}, \quad t \in[0, T] .
$$

Consequently the required a priori bounds are established and the results follows.

THEOREM 2.2. Let $f:[0, T] \times C \times R^{n} \rightarrow R^{n}$ be a continuous function. Assume that $\left(H_{1}\right)$ holds and moreover

$$
\begin{aligned}
& \left(H^{\prime}{ }_{2}\right) \text { There exist a continuous function } h: R^{+} \rightarrow R^{+} a \\
& \text { constant } N>0 \text { and nonnegative constants } q_{t}, \quad \imath=1, \ldots, 6 \\
& \text { such that } \\
& \begin{array}{r}
<v, f(t, u, v)>\leq h\left(|v|^{2}\right)\left(q_{1}|u(0)|^{2}+q_{2}|v|^{2}\right. \\
+q_{3}|u(0)||v|+q_{4}|u(0)| \\
\left.+q_{5}|v|+q_{6}\right)
\end{array}
\end{aligned}
$$

for all $t \in[0, T], v \in R^{n}$ and $u \in C$ with $|u| \leq M$ and

$$
\int_{\frac{4^{2}}{T}}^{N} \frac{d s}{h(s)} \geq 2 Q
$$

where

$$
Q=q_{1}(1+\sqrt{T})^{2} M^{2}+q_{2} M^{2}+q_{3}(1+\sqrt{T}) M^{2}+q_{4}(1+\sqrt{T}) \sqrt{T} M+q_{5} \sqrt{T} M+q_{6} .
$$

Then the BVP $(\mathrm{E})-(\mathrm{BC})$ has at least one solution. 
PROOF. Since the first part of the proof is similar to that of Therem 2.1 we give only the part of the proof which concens the a priori bound of ma.r $\left\{\left|r^{\prime}(t)\right|: 0 \leq t \leq T\right\}$. Taking the inner product of $\left(E_{\lambda}\right)$ with $x^{\prime}(t)$ we have by $\left(H_{2}^{\prime}\right)$

$$
\begin{aligned}
\left.\left|\frac{d}{d t}\right| x^{\prime}(t)\right|^{2} \mid \leq 2 h\left(| x ^ { \prime } ( t ) | ^ { 2 } \left(q_{1}|\cdot x(t)|^{2}+q_{2}\left|\cdot x^{\prime}(t)\right|^{2}\right.\right. & +q_{3}\left|\cdot r(t) \| \cdot r^{\prime}(t)\right|+q_{1}|r(t)| \\
& \left.+q_{5}\left|r^{\prime}(t)\right|+q_{6}\right)
\end{aligned}
$$

or

$$
\left|\frac{d}{d t} \int_{0}^{\left|r^{\prime}(t)\right|^{2}} \frac{d s}{h(s)}\right| \leq 2\left(q_{1}|\cdot r(t)|^{2}+q_{2}\left|\cdot r^{\prime}(t)\right|^{2}+q_{3}|\cdot x(t)|\left|\cdot r^{\prime}(t)\right|+q_{1}|\cdot r(t)|+q_{5}\left|\cdot r^{\prime}(t)\right|+q_{6}\right)
$$

Integrating the above inequality, and using the C'auchy. Schwarz inecquality we get

$$
\begin{aligned}
\int_{0}^{\left|x^{\prime}(t)\right|^{2}} \frac{d s}{h(s)} & \leq \int_{0}^{\left|s^{\prime}\left(t_{0}\right)\right|^{2}} \frac{d s}{h(s)}+\left\{q_{1} \int_{0}^{T}|\cdot r(t)|^{2} d t+q_{2} \int_{0}^{T}\left|r^{\prime}(t)\right|^{2} d t\right. \\
& \left.+q_{3} \int_{0}^{T}\left|\cdot r(t) \| \cdot r^{\prime}(t)\right| d t+q_{1} \int_{0}^{T}|\cdot r(t)| d t+q_{5} \int_{0}^{T}\left|\cdot r^{\prime}(t)\right|^{2} d t+q_{n}\right\} \\
& \leq \int_{0}^{\left|s^{\prime}\left(t_{0}\right)\right|^{2}} \frac{d s}{h(s)}+2\left\{q_{1}\|\cdot r\|^{2}+q_{2}\left\|\cdot r^{\prime}\right\|^{2}+q_{s}\|\cdot r\|\left\|\cdot r^{\prime}\right\|\right. \\
& \leq \int_{0}^{\left|s^{\prime}\left(t_{0}\right)\right|^{2}} \frac{d s}{h(s)}+2 Q \\
& \leq \int_{0}^{\frac{M^{2}}{T}} \frac{d s}{h(s)}+\int_{\frac{M^{2}}{T}} \frac{d s}{h(s)}=\int_{0}^{N} \frac{d s}{h(s)} .
\end{aligned}
$$

Hence

$$
\left|x^{\prime}(t)\right| \leq \sqrt{N}, \quad t \in[0, T]
$$

which completes the proof.

Now we present some examples to illustrate how the above results may be used to yield existence of solutions of specific boundary value problems.

EXAMPLE 2.3. We consider the following BVP

$$
\begin{gathered}
x^{\prime \prime}(t)+g(t) x(t) F\left(t, x_{t}, x^{\prime}(t)\right)=0, \quad t \in[0,1] \\
x_{0}-x^{\prime}(0)=\phi \\
x(1)+x^{\prime}(1)=2
\end{gathered}
$$

where $g:[0,1] \rightarrow R$ is a continuous and positive function and $F:[0,1] \times C \times R^{n} \rightarrow R^{n}$ a bounded function with bound $K$.

Here $f(t, u, v)=g(t) u(0) F(t, u, v), T=1, \alpha_{0}=\alpha_{1}=\beta_{0}=\beta_{1}=1$ and $\eta=2$.

Then we have

$$
<u(0), f(t, u, v)>=g(t) u^{2}(0) F(t, u, v) \leq g_{0} \Pi|u(0)|^{2}
$$

i.e $\left(H_{1}\right)$ holds with $A=g_{0} K, g_{0}=\max \{g(t): t \in[0,1]\}$ and $B=0$. Without loss of generality we can choose the functions $g$ and $F$ in such a way that $A=1$.

By an easy calculation we find for $\phi(0)=2, k=2 \cdot c_{1}=1-\frac{1}{\sqrt{2}}=c_{0}, c=1-\frac{2+\sqrt{2}}{\pi^{2}} \simeq 3.7$. 
We remark also that

$$
\left\langle r \cdot f(t, u \cdot \cdot \cdot)>=g(t) u(0) F(t, u \cdot v) u \leq y_{0} \cdot M K|\cdot|=|\cdot|^{2}\left(\frac{. I}{\sqrt{|\cdot|^{2}}}\right)\right.
$$

This means that $\left(H_{2}\right)$ holds with $h(0)=\frac{M}{\sqrt{4}}$. (The condition $\int_{V^{2}}^{\prime} \frac{d y}{h(4)} \geq 2 M^{2}$ is obvious $)$.

Therefore the BIP (e)-(be) has at keast one solution by Therem 2.1.

EXAMPLE 2.4. It is easy to sec. as in the previous example, that the BVP

$$
\begin{gathered}
r^{\prime \prime}(t)+g(t) \cdot r(t) F\left(t . x_{1} . x^{\prime}(t)\right)+q(t)\left|r^{\prime}(t)\right|=0 . \quad t \in\left[0 . \frac{1}{9}\right] \\
r_{0}-x^{\prime}(0)=0 \\
r(1)+x^{\prime}(1)=\sqrt{2}
\end{gathered}
$$

where $q:\left[0, \frac{1}{9}\right] \rightarrow R$ is a continuous and positive function, has at least one solution, $f(0) \varphi(0)=\sqrt{2}$ and $B=1$.

\section{CONCLUDING REMARKS}

In [4] the BVP (E)-(BC) has been studied under the following conditions, which are briefly reproduced here.

$\left(A_{1}\right)$ There exist a constant $M>0$ such that $|u(0)|>M$ and $\langle u(0), u\rangle=0$ implies $<u(0), f(t, u, v)><0$ for all $t \in[0, T]$ and $v \in R^{n}$.

$$
\begin{aligned}
\left(A_{2}\right)<u(0), f(t, u, v) & >\leq k_{1} \mid v^{2}+k_{2} \\
& |<v, f(t, u, v)>| \leq\left(k^{\prime}{ }_{1}|v|^{2}+k^{\prime}{ }_{2}\right)|v| \text { for all } t \in[0, T], u \in C^{\prime} \text { and } v \in R^{n} .
\end{aligned}
$$

$\left(A_{3}\right)|f(t, u, v)| \leq q(t) \Omega(|v|)$ for all $t \in[0, T], u \in C$ and $v \in R^{n}$.

Let us add in the above list the assumptions $\left(H_{1}\right)$ and $\left(H_{2}\right)$ of Theorem 2.1 .

$\left(H_{1}\right)<u(0), f(t, u, v)>\leq A|u(0)|^{2}+B|u(0) \| v|$ for all $t \in[0, T], u \in C$ and $" \in R^{n}$.

$\left(H_{2}\right)<v, f(t, u, v)>\leq h\left(|v|^{2}\right)|v|^{2}$ for all $t \in[0, T], u \in C$ and $v \in R^{n}$.

We also remind that:

The BVP (E)-(BC) has at least one solution if

$\left(A_{1}\right)$ and $\left(A_{2}\right)$ hold, [4. Th. 4.1]

$\left(A_{1}\right)$ and $\left(A_{3}\right)$ hold, [4. Th. 4.2]

$\left(H_{1}\right)$ and $\left(H_{2}\right)$ (or $\left(H^{\prime}{ }_{2}\right)$ ) hold, Th. 2.1 (or Th. 2.2)

The following questions are immediately arisen.

Has the BVP (E)-(BC) a solution if

1) $\left(A_{1}\right)$ and $\left(H_{2}\right)$ (or $\left.\left(H^{\prime}{ }_{2}\right)\right)$ hold ?

2) $\left(H_{1}\right)$ and $\left(A_{2}\right)$ hold ?

3) $\left(H_{1}\right)$ and $\left(A_{3}\right)$ hold ?

The answer in all of the above questions is positive. Indeed the cases 2) and 3) are obvious, since every one of conditions $\left(H_{1}\right),\left(A_{2}\right)$ and $\left.A_{3}\right)$ gives independently a priori bound on $x$ or $x^{\prime}$.

Some comments are needed for the case 1$)$. By taking the inner product of $\left(E_{\lambda}\right)$ with $x(t)$, integrating by parts over $[0, T]$ and using $\left(A_{1}\right)$ we get

$$
\left\|x^{\prime}\right\|^{2}+\frac{\alpha_{0}}{\alpha_{1}}|x(0)|^{2}+\frac{\beta_{0}}{\beta_{1}}|x(T)|^{2} \leq \frac{|\phi(0)|}{\alpha_{1}}|x(0)|+\frac{|\eta|}{\beta_{1}}|x(T)|
$$


or

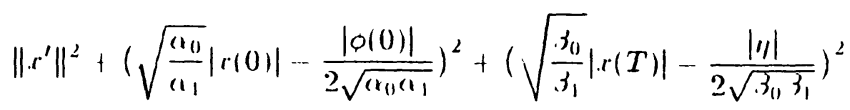

$$
\begin{aligned}
& \leq \frac{|\phi(0)|^{2}}{4 a_{0} \alpha_{1}}+\frac{\left|\eta_{1}\right|^{2}}{4 \cdot 3_{0} \cdot 3_{1}} \text {. }
\end{aligned}
$$

This implics the existence of the bound $M$ and the rest of the proof is cssentially the same as in Theorem 2.1 .

We summarize the above discussion in the following

THEOREM 3.1. The BVP (E)-(BC) has at least one solution if one of the following pairs of conditions holds:
1) $\left(A_{1}\right)$ and $\left(A_{2}\right)$
2) $\left(A_{1}\right)$ and $\left(A_{3}\right)$
3) $\left(A_{1}\right)$ and $\left(H_{2}\right)$ (or $\left(H^{\prime}{ }_{2}\right)$
4) $\left(H_{1}\right)$ and $\left(A_{2}\right)$
5) $\left(H_{1}\right)$ and $\left(A_{3}\right)$
6) $\left(H_{1}\right)$ and $\left(H_{2}\right)$ (or $\left(H^{\prime}{ }_{2}\right)$

\section{REFERENCES}

[1] J. Dugundji and A. Granas, Fixed Point Theory, Vol. I, Monographic Matematycane, PNW Warsawa, 1982 .

[2] D. Hai, Existence and uniqueness of solutions for a nonlinear second order differential equation in Hilbert space, Pror. Edinburgh Math. Soc. 33 (1990), 89-95

[3] J. Hale, "Theory of Functional Differential Equations" Springer-Vorlag, New York, 1977.

[4] S. Ntouyas, Y. Sficas and P. Tsamatos, Boundary value problems for functional differential equations, Technical Report No 187, (1991), University of Ioannina, Greece.

[5] D. O'Regan, Boundary value problems for second and higher order differential equations, Proc. Amer. Math. Soc. 113 (1991), 761-775. 


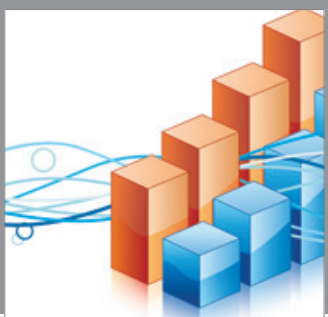

Advances in

Operations Research

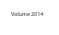

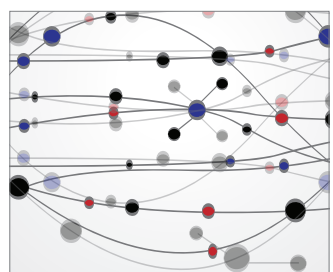

\section{The Scientific} World Journal
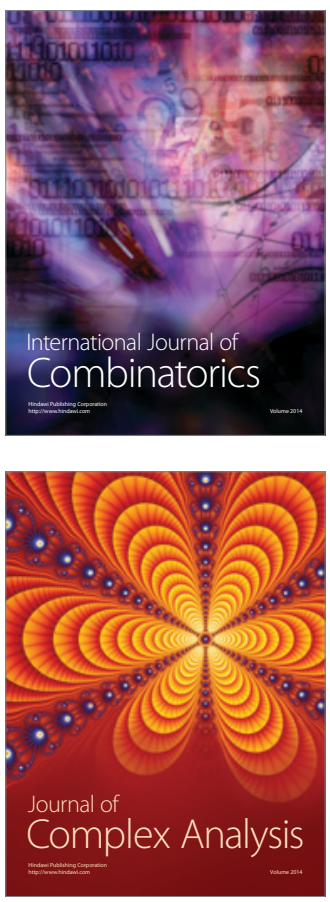

International Journal of

Mathematics and

Mathematical

Sciences
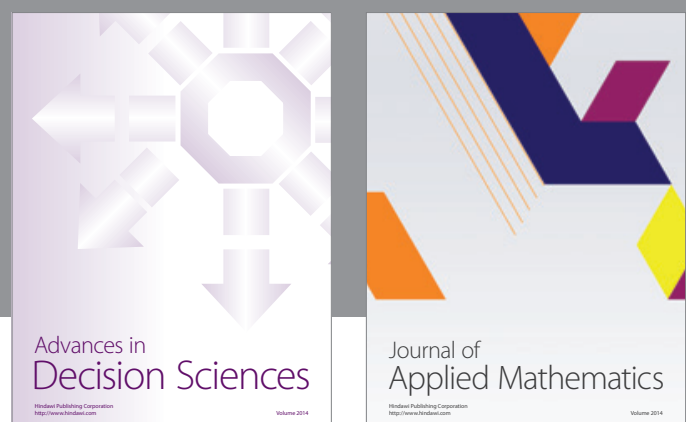

Journal of

Applied Mathematics
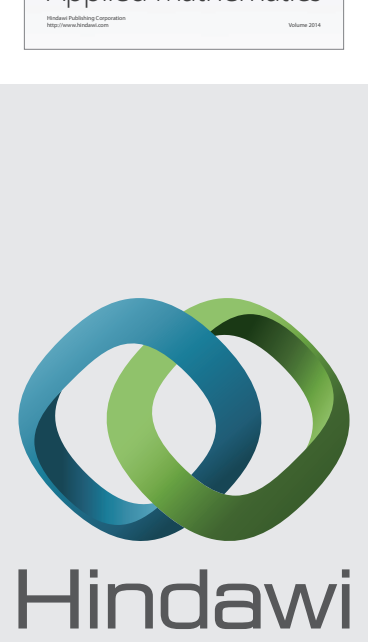

Submit your manuscripts at http://www.hindawi.com
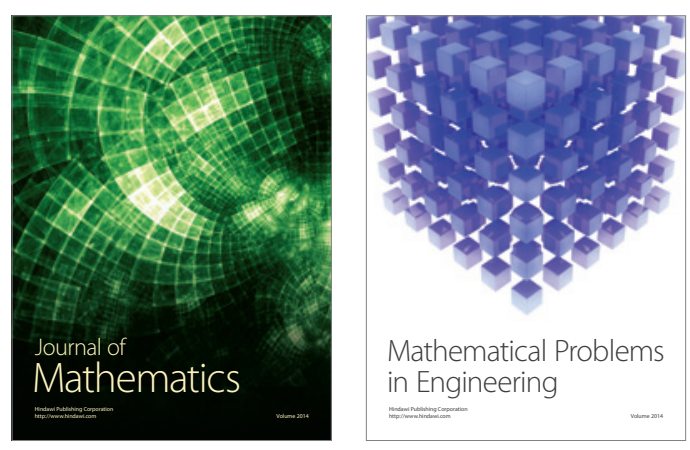

Mathematical Problems in Engineering
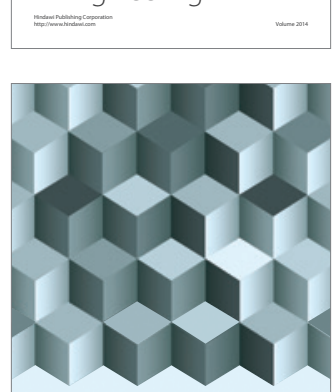

Journal of

Function Spaces
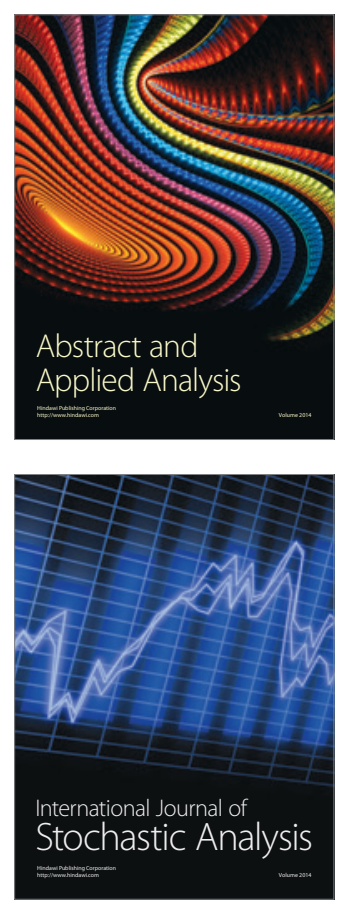

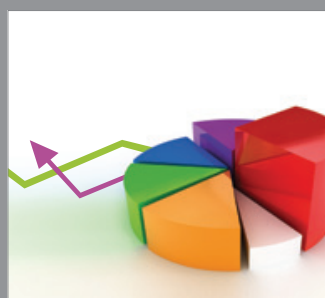

ournal of

Probability and Statistics

Promensencen
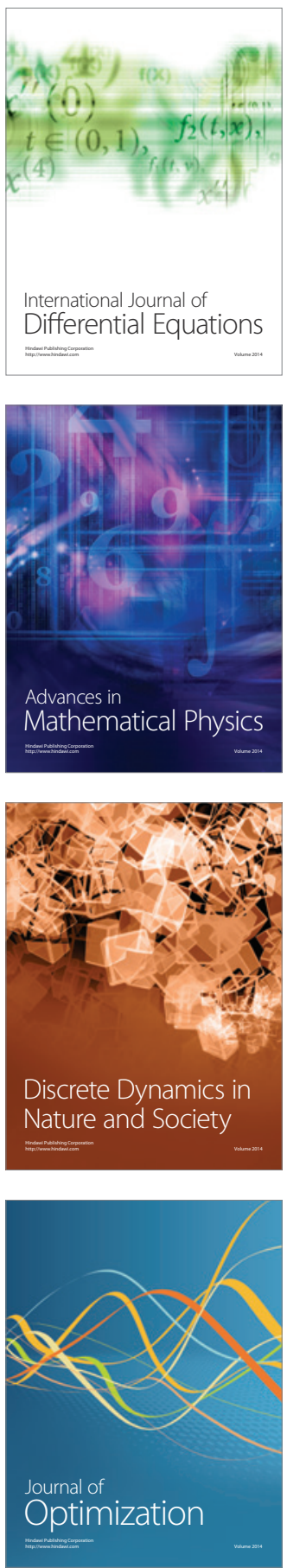